\title{
The arl locus positively regulates Staphylococcus aureus type 5 capsule via an $m g r A$-dependent pathway
}

\begin{abstract}
Correspondence
Chia Y. Lee

clee2@uams.edu
\end{abstract}

Received 30 May 2006

Revised 9 July 2006

Accepted 17 July 2006

\author{
Thanh T. Luong and Chia Y. Lee
}

Department of Microbiology and Immunology, University of Arkansas for Medical Sciences, Little Rock, AR 72205, USA

\begin{abstract}
Most clinical Staphylococcus aureus strains produce either type 5 or type 8 capsular polysaccharides. The production of these capsules is influenced by various environmental factors. To study the regulation of capsule, Tn551 transposon mutagenesis and transcriptional reporter gene fusion were employed to identify several putative regulatory loci that influenced capsule gene expression. One of these, the arl locus, was chosen for further analysis. Tn551 was found to insert within the coding region (near the translational start site of the arlR gene). ArIR, along with ArIS, forms a two-component system that has been previously shown to affect autolysis and production of several secreted proteins. Phenotypic analyses of the arlR-specific mutant and gene fusion analyses showed that arlR activated capsule production at the transcriptional level. However, gel mobility shift assays did not support activation of the capsule genes by direct ArIR binding to the primary cap5 promoter region upstream of the operon. In contrast, it was found that arl activated $m g r A$, an activator for capsule production, whereas $m g r A$ did not have a significant effect on $\operatorname{ar} / R$. Genetic studies supported the notion that $\operatorname{arlR}$ functions upstream of $m g r A$ with respect to the regulation of capsule production, although gene fusion studies indicated that arl could also regulate capsule independently from $m g r A$. Collectively, the results suggest that arl positively regulates capsule production at the transcriptional level primarily through an $m g r A$-dependent pathway.
\end{abstract}

\section{INTRODUCTION}

Staphylococcus aureus produces several virulence factors which are essential for its pathogenicity. Among these are extracellular toxins and enzymes, and cell-wall-associated molecules. Capsular polysaccharide (CP) is one of the surface molecules produced by most $S$. aureus strains. Several serotypes of CPs have been reported to date but most human clinical $S$. aureus isolates produce capsule of either serotype 5 (CP5) or serotype 8 (CP8). The role of these capsules in $S$. aureus virulence has recently been investigated in different laboratories using molecularly characterized strains and different animal models. These studies provide compelling evidence that $\mathrm{CP} 5$ and $\mathrm{CP} 8$ are important antiphagocytic virulence factors (for reviews see Lee \& Lee, 2006; O’Riordan \& Lee, 2004). Additionally, staphylococcal capsule was recently implicated as one of the few extracellular factors required for virulence in Caenorhabditis elegans (Bae et al., 2004).

The cap5 and cap 8 operons required for the synthesis of CP5 and CP8 are allelic (Sau et al., 1997a). Twelve of the 16 genes are virtually identical between the cap5 and cap 8 operons, which explains why CP5 and CP8 have almost identical

Abbreviation: $\mathrm{CP}$, capsular polysaccharide. trisaccharide-repeating units that differ only in the location of the $O$-acetylation and the position linking monosaccharides. Molecular characterization of the cap 8 locus indicates that all 16 genes are transcribed as a large transcript from the primary promoter upstream of the first gene. Several internal promoters are also present but these are much weaker than the primary promoter (Sau et al., 1997b). A $10 \mathrm{bp}$ inverted repeat located upstream of the -35 sequence of the primary cap 8 promoter has been shown to be required for full expression of $\mathrm{CP} 8$, suggesting that a DNA-binding regulator is involved in the control of capsule production (Ouyang et al., 1999). The promoter regions between the cap5 and cap8 operons of several strains are also highly similar, suggesting that the two capsules are regulated similarly (Herbert et al., 2001; Sau et al., 1997a).

Several regulatory genes which globally control the expression of many virulence determinants of $S$. aureus have been identified. These regulators appear to form a complex network of regulation to coordinately control the virulence gene expression (for reviews see Bronner et al., 2004; Cheung et al., 2004; Novick, 2003). Previously, we showed that agr had a significant effect on capsule production (Luong et al., 2002). Recently, we found that $m g r A$, also known as norR (Truong-Bolduc et al., 2003) or rat (Ingavale et al., 2003), affected capsule production (Luong et al., 
2003). In this study, we employed Tn551 mutagenesis to identify several putative regulatory loci that influenced CP5/ 8 production. We chose to focus on $\mathrm{arl}$, a locus which has previously been shown to regulate autolysis and some secreted proteins (Fournier \& Hooper, 2000; Fournier et al., 2001), and showed that the locus positively regulated CP5/8 expression at the transcriptional level mainly via an $m g r A-$ dependent pathway.

\section{METHODS}

Bacterial strains and plasmids. S. aureus strains and plasmids used in this study are listed in Table 1. S. aureus strain RN4220 was used as the recipient for electroporation of the constructed plasmids. Transductions between $S$. aureus strains were carried out using bacteriophage 52A. S. aureus strains were cultivated on tryptic soy agar (Difco). Electroporation in S. aureus was carried out by the procedure of Kraemer \& Iandolo (1990). Nitrocefin was purchased from Becton Dickinson Microbiology Section.

Transposon mutagenesis and identification of Tn551 insertion sites. Random transposition was carried out by temperature shift using strain COL(pI258repA36). A library of 20000 Tn551insertion mutants was collected and divided into 16 separate pools, and each pool was transduced with pCL8074. The transductants were then screened for increased or decreased yellow colour by spraying the colonies with $0.5 \mathrm{M}$ catechol as described by Zukowski et al. (1983). To identify Tn551 insertion sites, chromosomal DNA with Tn551 insertion was digested with Sau3A, ligated with T4 DNA ligase and amplified by inverse PCR using two inverse PCR primers (Tn917R and Rn917L; Table 2) as previously described (Luong et al., 2003). The amplified fragments were cloned into pGEMT-easy vector (Promega) and sequenced.

Plasmid construction. Plasmid pCL96 was constructed from pCL95 (Luong \& Lee, 2002) by reversing the orientation of tet (pT181) and ori (pE194) with respect to the rest of the plasmid. Plasmids pLL33 and pLL35 were constructed by ligating the blaZ reporter gene and the multiple cloning sites from pWN1819 and pSA3800, respectively, to pCL96. Plasmid pCL15 was derived from pSI-1 (Yansura \& Henner, 1984) by removing the EcoRI site (endfilling), adding the ampicillin-resistance gene from pBluescript at the $B a m H I$ site (which also destroyed the BamHI site) and replacing the cloning site with the multiple cloning site of pUC18. To construct Pcap5:: blaZ reporter fusions, a $627 \mathrm{bp}$ fragment containing the cap5 promoter was amplified from Newman chromosomal DNA using primers cp8bla.f and cp8bla.r. To clone the $m g r A$ gene under the control of Pspac in pCL15, a 570 bp fragment containing the $m g r A$ gene without the promoter was amplified using primers mgr74 and mgr75 and cloned into the HindIII and XbaI sites of pCL15. The same method was used to clone an $820 \mathrm{bp}$ fragment containing the arlR gene in pCL15 using primers arlR19 and arlR20. To clone the arlR gene for complementation, a PCR fragment was amplified from Newman chromosomal DNA using arlR17 and arlR18 and then cloned into pCL96 to form pTL3287.

Table 1. S. aureus strains and plasmid vectors

\begin{tabular}{|c|c|c|}
\hline Strain or plasmid & Relevant characteristics & Reference \\
\hline \multicolumn{3}{|l|}{ S. aureus } \\
\hline Becker & CP8 strain & \\
\hline COL & CP5 strain & \\
\hline Newman & CP5 strain & \\
\hline RN4220 & $8325 \mathrm{r}^{-}$ & Kreiswirth et al. (1983) \\
\hline CYL1164 & Newman $\Delta a r l R$ & This study \\
\hline CYL815 & Newman arlR:: Tn551 & This study \\
\hline CYL1050 & Newman $\Delta m g r A::$ cat & This study \\
\hline CYL1206 & Newman $\triangle m g r A:$ : cat $\Delta a r l R$ & This study \\
\hline CYL1460 & Newman $\Delta m g r A$ & This study \\
\hline \multicolumn{3}{|l|}{ Plasmid } \\
\hline pI258rep36 & Tn551 delivery plasmid & Novick et al. (1979) \\
\hline pCL52.2 & Temperature-sensitive cloning vector & Sau et al. (1997b) \\
\hline pCL96 & Cloning vector & This study \\
\hline pSA3800 & Transcriptional blaZ fusion vector & Novick et al. (1989) \\
\hline pWN1819 & Translational balZ fusion vector & Wang et al. (1987) \\
\hline pLL33 & Translational blaZ fusion vector & This study \\
\hline pLL35 & Transcriptional blaZ fusion vector & This study \\
\hline pCL15 & Cloning vector containing Pspac promoter & This study \\
\hline pCL997 & Pcap 5:: blaZ transcriptional fusion in pSA3800 & This study \\
\hline pCL998 & Pcap5:: blaZ translational fusion in pWN1819 & This study \\
\hline pAM3175 & Pcap5:: blaZ translational fusion in pLL33 & This study \\
\hline pAM3176 & Pcap5:: blaZ transcriptional fusion in pLL35 & This study \\
\hline pTL3287 & pCL96 containing arlR gene & This study \\
\hline pCL8074 & Pcap8: : xylE transcriptional fusion in pLC4 & Ouyang et al. (1999) \\
\hline pCL3292 & pCL15 containing $m g r A$ & This study \\
\hline pTL3573 & pCL15 containing arlR & This study \\
\hline
\end{tabular}


Table 2. Primers for PCR

\begin{tabular}{|ll|}
\hline Primer & \multicolumn{1}{c|}{ Sequence } \\
\hline Tn917R & GCTATGCTCGAGTGAGTACG \\
Tn917L & CACAATAGAGAGATGTCACC \\
arlR1 & CATATGACGCAAATTTTAATAG \\
arlR2 & GGATCCTCATCGTATCACATAC \\
arlR3 & GGTGTACATTATCCAACAGA \\
arlR4 & AGGTGTAGATTGTTGTTGTC \\
arlR5 & GATTGAAACAGTTCGTGGCG \\
arlR6 & TAGATGTCAGATCCGTATCA \\
arlR17 & CTAATTTAAAAAGCAACTATTGT \\
arlR18 & TTACGTTTTGTCATCGTATCACAT \\
arlR19 & ATAAAGCTTCGTAATATGAGGTGTACAAATG \\
arlR20 & ATATCTAGAGCTCACTATTATGCAGTGTA \\
cp8bla.f & CTGCAGAGCTCGCATTTGAAGATCA \\
cp8bla.r & GGATCCCCTTAGTTTGATTCACTAAA \\
cp8gs6 & CTACTTTAGAGTATAATTATTTTTAATTTC \\
cp8gs15 & TAAAAATTTTCATTAAAATTG \\
mgr74 & AAGCTTTAGTATGTCTGGAGGAGAAC \\
mgr75 & TCTAGAAACTAACAAGCTTACGCGTGC \\
SG16S1 & CGTGCTACAATGGACAATACAAA \\
SG16S2 & ATCTACGATTACTAGCGATTCCA \\
SGhu1 & GCTGGGATATCAATTTCTTTACC \\
SGhu2 & ATCCAAAACTCACTTGCTAAAGG \\
SGarlR1 & TTACGGTGCAGACGATTATATAG \\
SGarlR2 & TACCGTTGACATCGATAATATCC \\
SGmgrA1 & AGACAAGTTAATCGCTACTACTC \\
SGmgrA2 & CTAATTCAGTTACGACTTTCTTG \\
SGcap8A1 & ACTAAGGGTGACAATCCTCAG \\
SGcap8A2 & AAGTCCTTTGACACCTCATCTA \\
\hline
\end{tabular}

Strain construction. To construct the Newman $\Delta \operatorname{arl} R$ mutant, the upstream and downstream fragments of the target region to be replaced were amplified by PCR using two sets of primers (arlR3 and arlR4 for upstream fragment and arlR5 and arlR6 for downstream fragment) and sequence-verified. The two fragments were cloned into pCL52.2 and used for allele replacement as described previously (Lin et al., 1994). The mutant (CYL1164) with a $383 \mathrm{bp}$ internal deletion of the $\operatorname{arl} R$ gene without any replacing antibioticresistance marker was obtained and verified by PCR.

The Newman $\Delta m g r:$ :cat strain (CYL1050) was constructed by transducing $\triangle m g r A:$ :cat from CYL1040 (Luong et al., 2003) to strain Newman. To construct the Newman $\Delta m g r A$ strain (CYL1460), the plasmid used for the construction of CYL1040 was digested with ApaI to delete the cat gene. The resultant plasmid was transferred to CYL1050 for allele replacement as described by Lin et al. (1994) except that the clones were screened for the loss of chloramphenicol resistance. The resultant strain CYL1460 was confirmed by PCR with appropriate primers. To construct $m g r A$ arlR double mutant CYL1206, the $\Delta m g r A$ : : cat from CYL1040 was transduced to CYL1164.

RNA extraction and mRNA quantification. These were carried out as described previously (Luong et al., 2002). For quantification of mRNA by real-time RT-PCR, gene-specific primers (SG16S1, SG16S2, SGhu1, SGhu2, SGarl1, SGarl2, SGmgrA1, SGmgrA2, SGcap8A1, SGcap8A2; Table 2) were used to amplify 100-200 nucleotide fragments of the target genes (Table 2). DNase I-treated RNA was incubated with the SuperScript III platinum SYBR Green One-Step qRT-PCR master mix (Invitrogen) and the reaction was carried out using the ABI Prism 7300 Detection system (Applied Biosystems). No-template reactions were included as negative controls. A negative control without reverse transcriptase in the reaction was performed to exclude the possibility of DNA contamination. Standard curves were established to confirm that the primers amplified at the same rate. The $16 \mathrm{~S}$ rRNA and/or the $h u$ gene was used for normalization of the target mRNA. Relative expression levels were determined by using the protocol for the standard curve method (Applied Biosystems user bulletin no. 2).

Gel mobility shift assay. The $\operatorname{arl} R$ gene was amplified by primers arlR1 and arlR2, cloned into the NdeI and BamHI sites of pET15b (Novagen) and used for purifying $\mathrm{His}_{6}$-ArlR fusion protein by nickel-affinity column chromatography following the manufacturer's directions (Novagen). A 138 bp fragment containing the promoter of the cap5 operon (from +4 to -135 with respect to the transcriptional start site) was amplified by PCR using primers cp8gs6 and cp8gs15, purified using a Qiagen PCR purification kit, end-labelled and used in gel mobility shift assay as previously described (Ouyang et al., 1999). Phosphorylation of $\mathrm{His}_{6}$-ArlR was carried out by incubating $2 \cdot 3 \mu \mathrm{g}(86 \cdot 7 \mathrm{pmol})$ purified $\mathrm{His}_{6}-\mathrm{ArlR}$ at $37^{\circ} \mathrm{C}$ for $2 \mathrm{~h}$ in $10 \mu \mathrm{l}$ reaction buffer containing $50 \mathrm{mM}$ Tris/ $\mathrm{HCl}(\mathrm{pH} 8 \cdot 0), 10 \mathrm{mM}$ $\mathrm{MgCl}_{2}, 2 \mathrm{mM}$ DTT and $30 \mathrm{mM}$ acetyl phosphate. Successful phosphorylation was determined by detecting a shifted band in an $8 \%$ non-denatured PAGE system.

Other tests. $\beta$-Lactamase activity was assayed by the nitrocefin method as described previously (Luong et al., 2002). CP5 production was quantified as described by Luong et al. (2003).

Statistical analysis. Data from reporter gene fusion analyses were assessed using a paired Student's $t$ test for comparing two samples. $P$ values of $<0.05$ were considered statistically significant.

\section{RESULTS}

\section{Screening of Tn551 mutants}

To identify potential genes involved in capsule regulation, our strategy was to introduce plasmid pCL8074 carrying the Pcap 8: : xylE fusion into a mutant pool of an S. aureus strain and screen the colonies for mutants with altered XylE activities, which could be detected by colour change when the colonies were sprayed with the XylE substrate. A colour difference between a mutant and the wild-type would suggest that the mutation affects cap8 gene transcription. Plasmid pCL8074 was used previously for studying cap8 promoter activity in our laboratory (Ouyang et al., 1999) but its use for monitoring the activity in isolated colonies had not been tested. We therefore transferred the plasmid into strains Becker, Newman and COL for testing XylE activity. We found that COL(pCL8074) exhibited moderate intensity of yellow colour, strain Becker(pCL8074) exhibited high intensity and Newman(pCL8074) exhibited low intensity. We chose strain COL for further transposon mutagenesis for screening potential regulatory genes because of the possibility of obtaining both classes of mutation, activator and repressor, in the same screen. Random transposition was carried out as described in Methods. Several putative mutants with reduced or enhanced yellow colour as compared to the wild-type were identified. Forty-four of these mutants were backcrossed by phage transduction to the parental strain COL and the phenotypes of 17 of them 
remained unchanged, indicating that the phenotypes are due to the Tn551 insertion and not to secondary mutations during mutagenesis.

The chromosomal regions flanking Tn551 insertion sites in the 17 mutants identified above were amplified by inverse PCR, which yielded 12 different patterns according to the sizes of the amplified fragments. The fragments representing each of the 12 patterns were subjected to DNA sequencing. BLAST searches of the sequenced DNA fragment against the genomes of strains 8325 and N315 showed that the insertion sites in these mutants were within the coding region of $\operatorname{arl} R$, $s b c D, s b c C, c l p C, \mathrm{~N} 315$ SA1288, N315 SA1708 and N315 SA0816. A single insertion site was found in each gene, except that there were six different insertion sites within $s b c D$ gene. The insertions within $s b c C, s b c D$ and $c l p C$ resulted in increased XylE activities, whereas insertions in other genes resulted in decreased XylE activities.

\section{arlR is an activator for capsule production}

To study whether the genes identified above are involved in capsule regulation, we chose to focus on $\operatorname{arl} R$, the response regulator of the arlRS two-component regulatory system. The arlRS system has been shown to be involved in regulation of several virulence genes in S. aureus (Fournier \& Hooper, 2000; Fournier et al., 2001). The two genes form an operon, and mutations in each gene have been shown to result in the same phenotype (Fournier et al., 2001). To further confirm that the arl locus is involved in the regulation of capsular production, we transduced the Tn 551 insertion in the $\operatorname{arlR}$ gene (at between 66 and $67 \mathrm{bp}$ from the translational start site) from strain COL to strain Newman, which produces type 5 capsule. The resultant strain (CYL815) produced much less capsule than the wild-type strain when the capsule was isolated from whole cell pellet or from the supernatant (Fig. 1a). We then constructed an arlR-deletion mutant (CYL1164) in which the $383 \mathrm{bp}$ internal portion of the gene was deleted by allele replacement. As shown in Fig. 2, similar to the Tn551 mutant CYL815, the mutant CYL1164 produced an almost undetectable amount of CP5 as compared to the wild-type strain. To confirm that the mutant phenotype is due to the deletion of $\operatorname{arl} R$, we complemented CYL1164 with pTL3287, carrying a PCRamplified 739 bp DNA fragment containing the intact arlR gene from strain Newman. As shown in Fig. 2, the capsule production of the CYL1164(pTL3287) was restored to the wild-type level. Since the cloned fragment contained only the $\operatorname{arlR}$ gene without other ORFs, the results indicated that the mutation in arlR, not other secondary mutations or polarity, affects capsule production in strain Newman. These results suggested that the arlR gene, and thus the arl locus, is involved in the activation of capsule production.

\section{arIR regulates cap5 gene expression at the transcriptional level}

To determine whether arlR regulates capsule production at the transcriptional level, we measured cap5-specific mRNA (a)

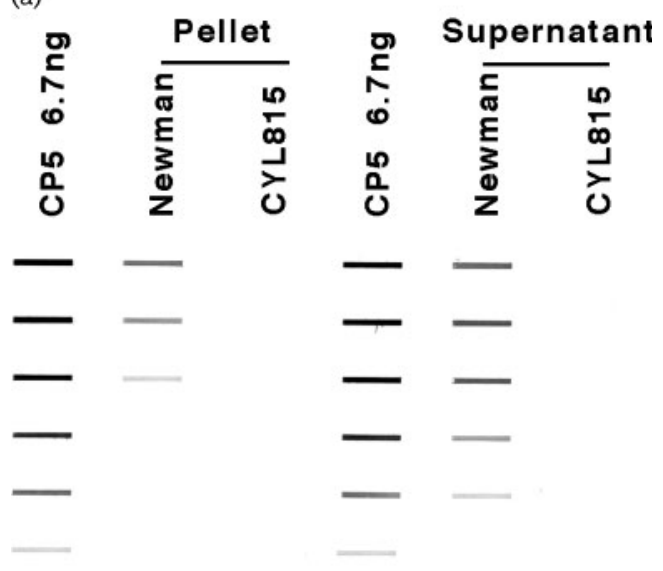

(b)

cap5D

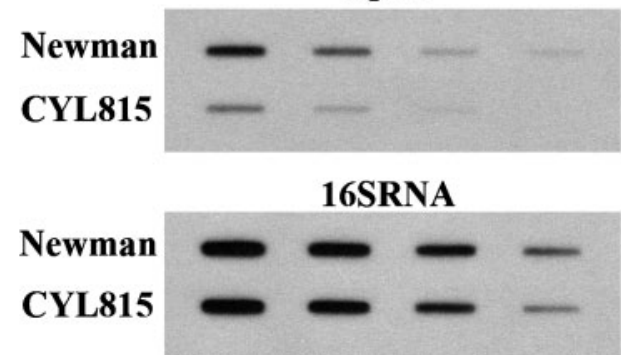

Fig. 1. Comparison of strains Newman and CYL815. (a) CP5 production. Equivalent amounts of samples adjusted according to $\mathrm{OD}_{660}$ were taken from overnight cultures (at $\mathrm{OD}_{660} 7 \cdot 5$ and $7 \cdot 7$ for Newman and CYL815, respectively) and processed as described in Methods. Samples were diluted threefold initially and further threefold serially diluted. The diluted samples (20 $\mu \mathrm{l}$ samples from pellet and $252 \mu \mathrm{l}$ samples from supernatant) were loaded to nitrocellulose membranes and incubated with rabbit anti-CP5 specific antibody. Threefold dilutions of CP5 standard with an initial content of $6.7 \mathrm{ng}$ in $20 \mu \mathrm{l}$ were loaded as controls. (b) cap5-specific mRNA. Total RNAs were purified from overnight cultures. The undiluted $(10 \mu \mathrm{g})$ and the twofold serially diluted samples were loaded to nitrocellulose membranes and probed with the cap5D gene (top panel). A duplicate set of samples was probed with the $S$. aureus $16 \mathrm{~S}$ rRNA gene (bottom panel) to indicate equal loading of the samples.

synthesis in the wild-type strain Newman and its isogenic arlR mutant CYL815 by RNA slot-blotting. Total RNAs were isolated from overnight cultures of Newman and CYL815 and hybridized either with the cap5D gene probe or with a 16S rRNA gene probe as a loading control. The results in Fig. 1(b) show that the amount of cap5 mRNA isolated from the mutant CYL815 was much less than that isolated from Newman. This result is similar to the assay of CP5 production described above, suggesting that arlR is an activator regulating $\mathrm{CP} 5$ production primarily at the transcriptional level.

To confirm the transcriptional regulation of cap 5 expression by $\operatorname{arl} R$ and to determine whether $\operatorname{arl} R$ can also have an 


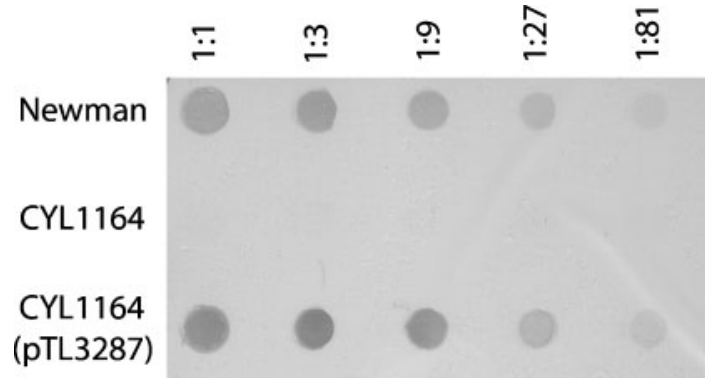

Fig. 2. CP5 from strains Newman, CYL1164 and the complemented strain CYL1164(pTL3287) from overnight cultures $\left(\mathrm{OD}_{660} 7 \cdot 3,6.9\right.$ and $7 \cdot 0$, respectively) was isolated as described in Methods. The undiluted $(30 \mu \mathrm{l})$ and the threefold serially diluted samples (indicated above the blot) were analysed by Western dot-blotting.

effect on CP5 production at the post-translational level, we constructed blaZ reporter gene fusions. The fusion plasmids were transduced into strain Newman and mutant strain CYL1164. As shown in Fig. 3, the Pcap5 activity in the arlR mutant was significantly less than that in the wild-type strain in both transcriptional and translational fusion assays at all time points. We also obtained similar results for BlaZ activities when the fusion plasmids were put into the Tn551 insertional mutants (data not shown). The fact that the BlaZ activities decreased in a similar pattern in both transcriptional and translational fusions suggests that the regulation of the cap 5 genes by arlR is controlled at the transcriptional level. However, we noted that translational fusions had a tenfold higher activity than the transcriptional fusions (Fig. 3). This difference could be due to the difference in ribosome-binding site of the two constructs.

\section{ArIR activates cap5 promoter indirectly}

To determine whether ArlR activates the cap 5 genes by direct binding, we performed gel mobility shift experiments by incubating a ${ }^{32}$ P-labelled DNA fragment of 138 bp from the promoter region of the cap 5 locus containing the $10 \mathrm{bp}$ inverted repeat previously shown to be required for cap5 activation (Ouyang et al., 1999) with increasing concentrations of purified $\mathrm{His}_{6}$-ArlR. We found a shifted band, which could be inhibited with 500-fold excess of unlabelled competitor DNA but not by an irrelevant DNA fragment (data not shown). However, the shifting could only be detected when the amount of ArlR was or exceeded $5 \mu \mathrm{M}$, suggesting that ArlR binds to the cap5 promoter region but only at a high concentration. Phosphorylation of ArlR with acetyl phosphate did not facilitate the binding (data not shown). Taking these results together, we concluded that ArlR might not regulate the capsule production by direct binding to the promoter region of the cap5 operon. Although the binding occurred under high concentrations of the protein, it was most likely not physiologically relevant.

\section{arlR regulates cap5 genes primarily through mgrA}

We have previously shown that $m g r A$ is a major activator of cap5(8) gene expression (Luong et al., 2003, 2006). Since we showed above that arl most likely did not regulate the cap5 genes directly, we speculated that it might regulate capsule production through $m g r A$. To test this possibility, we compared the mgrA mRNA produced from wild-type strain Newman and an arl deletion mutant from $18 \mathrm{~h}$ cultures using real-time RT-PCR. We found that the mgrA mRNA was reduced 18 -fold in the arlR mutant (data not shown), indicating that $m g r A$ is positively regulated by the arl locus. On the other hand, the amount of arlR mRNA from an mgrA mutant was similar to that from the wild-type, indicating
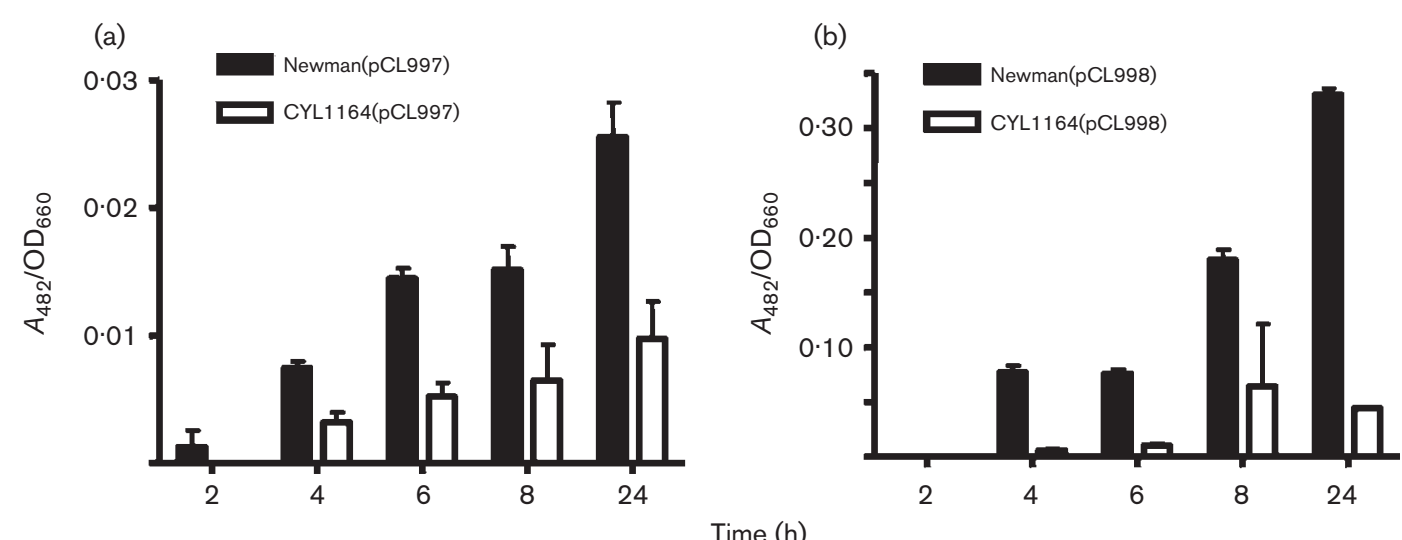

Fig. 3. BlaZ activities of cap $5:$ : blaZ transcriptional fusion in plasmid pCL997 (a) and of cap5:: blaZ translational fusion in plasmid pCL998 (b) in Newman and CYL1164 were analysed at 2, 4, 6, 8 and 24 h. The BlaZ activities are expressed as the ratio of $A_{482}$ of the enzymic reaction to the $\mathrm{OD}_{660}$ of the culture. The error bars indicate SD $(n=3)$. 
(a)

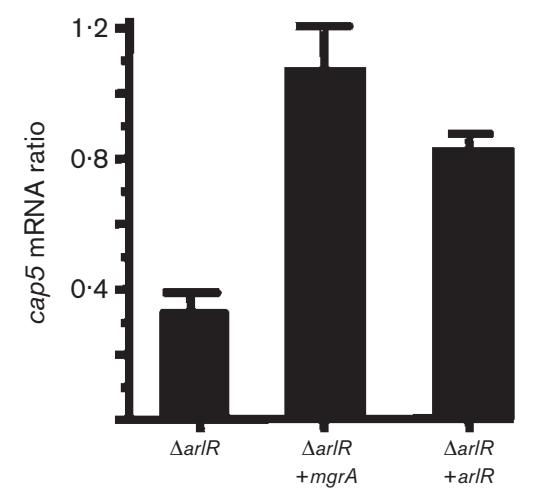

(b)

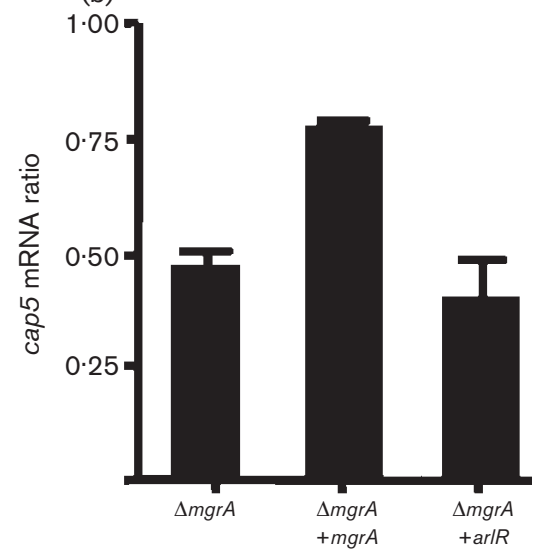

Fig. 4. Comparison of cap5 gene expression in $\triangle m g r A \quad$ [CYL1640(pCL15)], $\triangle m g r A+$ $m g r A$ [CYL1640(pCL3292)] and $\triangle m g r A+$ arlR [CYL1640(pTL3573)] (a), and in $\Delta a r l R$ [CYL1164(pCL15)], $\Delta$ arlR+mgrA [CYL1164(pCL3292)] and $\Delta a r l R+a r l R$ [CYL1164(pTL3573)] (b). Total RNAs were isolated from $18 \mathrm{~h}$ cultures in the presence of $1 \mathrm{mM}$ IPTG; the cap5 mRNAs were quantified by real-time RT-PCR and shown as a ratio to that of strain Newman (arbitrarily set at 1). The error bars indicate SD $(n=3)$. that mgrA has no significant effect on arl expression. These results suggest that arl may up-regulate cap5 through upregulation of mgrA.

To further confirm that arl functions upstream of $m g r A$ with respect to cap5 gene regulation, we measured the cap5 mRNA by real-time RT-PCR to determine whether overproducing MgrA can compensate cap5 expression in an arlR mutant and, conversely, whether overproducing ArlR can compensate cap5 expression in an mgrA mutant. The rationale for these experiments is that overproduction of MgrA, but not overproduction of ArlR, should restore the arl effect on capsule if arl is upstream of $m g r A$ in the regulatory circuit. On the other hand, a reverse effect should be detected if arl acts downstream of mgrA. To this end, we cloned the $m g r A$ or $\operatorname{arlR}$ gene under the control of the IPTGinducible promoter Pspac in pCL15. The results in Fig. 4(a) show that MgrA overproduction was able to restore the cap5 mRNA in the arl mutant to the wild-type level, whereas the results in Fig. 4(b) show that ArlR overproduction did not restore the cap 5 mRNA level in the $m g r A$ mutant. These results support the contention that arl functions upstream of the $m g r A$.

To determine whether arl regulates the cap5 genes solely through $m g r A$, we compared the transcriptional and translational blaZ reporter activities in Newman and the isogenic arl, $m g r A$ and double arlR mgrA mutants. Since the mgrA arlR double mutant strain CYL1206 was constructed with a chloramphenicol-resistance marker, the above Pcap5: : blaZ fusion plasmids pCL997 and pCL998, which have the same resistance marker, cannot be used. We therefore reconstructed the Pcap $5:$ : blaZ fusions in tetracyclineresistant pCL33 and pLL35 vectors, which resulted in transcriptional fusion plasmid pAM3175 and translational fusion plasmid pAM3176, respectively. As shown in Fig. 5,

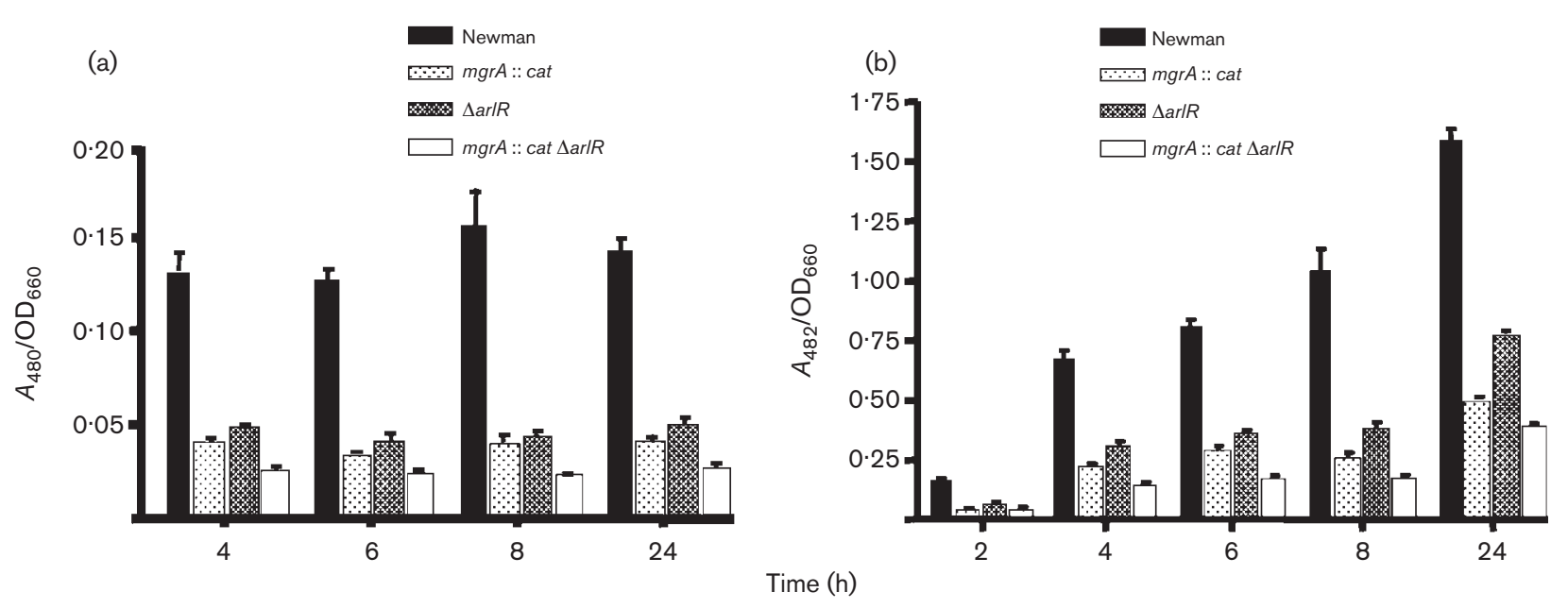

Fig. 5. The activities of cap5::blaZ transcriptional fusion in plasmid pAM3176 (a) and translational fusion in plasmid pAM3175 (b) in strains Newman, CYL1050 (Newman $\Delta m g r:: c a t)$, CYL1164 (Newman $\Delta a r l R$ ) and CYL1206 (Newman $\Delta m g r::$ cat $\Delta a r l)$ were analysed at $4,6,8$ and $24 \mathrm{~h}$. The BlaZ activities were expressed as the ratio of $A_{482}$ of the enzymic reaction to the $\mathrm{OD}_{660}$ of the culture. The error bars indicate SD $(n=3)$. 
for both transcriptional and translation fusions, mutations in $m g r A$ or $\operatorname{arl} R$ substantially reduced the cap5 promoter activity, and the double mutation reduced the activity more than the single mutations. The additional reduction in cap5 promoter activities at various time points in the double mutant as compared to that of the mgrA single mutant are statistically significant, with $P$ values (paired $t$ test) ranging from 0.0005 to 0.0135 for the transcriptional blaZ assays (Fig. 5a) and from 0.0029 to 0.021 for the translational blaZ assays (Fig. 5b). This reduction of the cap5 promoter activity resulting from arl mutation in the $m g r A$ mutant suggests that arl also regulates cap5 independently from $m g r A$. However, although the reduction is statistically significant, it is only moderate, suggesting that the $m g r A$ independent pathway(s) may only play a minor role in cap5 regulation.

\section{DISCUSSION}

S. aureus can cause a variety of diseases. Its pathogenicity depends on coordinated expression of various virulence genes. Regulation of these virulence genes is therefore critical in determining the outcome of an infection. In this study, we used transposon mutagenesis to generate random mutations in strain COL and identified several potential regulators that affect the production of capsule, one of the important virulence factors produced by most $S$. aureus strains. We identified several potential genes affecting capsule production. However, some known regulatory genes involved in capsule regulation, such as agr or $m g r$, were not identified, suggesting that the library is biased to some degree. Among the genes identified, we chose to focus on arl since this locus has been shown to be an important regulator affecting the expression of several virulence factors in $S$. aureus, including autolysin and various toxins (Fournier \& Hooper, 2000; Fournier et al., 2001), but how it regulates capsule has not been studied. Our results showed that arl positively controlled the production of capsule. These results are consistent with a recent gene profiling study showing that two of the cap 5 genes were upregulated by arl (Liang et al., 2005) and with a study showing that a natural deletion in the arlRS locus of the sequenced N315 strain affected CP5 production (Cocchiaro et al., 2006). The arl locus is composed of two genes, arlS and arlR, encoding the sensor and regulator, respectively, of a typical prokaryotic two-component regulatory system in which the sensor senses an environmental cue(s) and relates the information to the response regulator, usually, by phosphorylation (Klumpp \& Krieglstein, 2002). However, to date, no environmental cue has been reported to be mediated by ArlS. Several environmental factors, which include iron concentration, oxygen tension, carbon dioxide and growth media, have been reported to influence capsule production (Lee \& Lee, 2006; O'Riordan \& Lee, 2004). The environmental control of virulence gene expression in S. aureus is an understudied area. It would be of interest to determine if the signal of any of these environmental factors affecting capsule production is transduced through the arl system.
The expression of virulence genes in S. aureus is controlled by very complicated regulatory circuitry (Bronner et al., 2004; Cheung et al., 2004; Novick, 2003) in which various regulators form a complex network to coordinately regulate different virulence genes. With regard to capsule, we have previously shown that both agr and mgr positively regulate capsule production mostly at the transcriptional level, whereas sarA does not play a role in transcriptional regulation but may have a minor effect at the post-translational level (Luong et al., 2002, 2003). In this study, we found that arl played an activator role, exerting its effect at the transcriptional level, similar to that of agr and mgr. However, how arl regulates its target genes has not been studied. Since arl is a two-component system, it is likely that ArlR binds some target genes directly. Although our gel mobility shift experiments showed that both the unphosphorylated and phosphorylated forms of ArlR could bind to the cap5 promoter region, the concentrations of the protein at which the binding occurred were most likely too high to be within the physiological condition. Hence the binding may not be biologically significant. Our results, therefore, suggest that ArlR most likely does not regulate capsule production by direct binding to the cap 5 promoter region.

As the gel-shift experiments indicated that arl probably regulates capsule through other regulators, to test this possibility we investigated whether arl affected $m g r A$ expression. We found that $m g r A$ expression was drastically reduced (18-fold) in an arlR deletion mutant, whereas mgrA mutation did not affect the expression of arlR, indicating that arl activates $m g r A$ but not the opposite. We also showed that $m g r A$ expressed from an inducible promoter could override the arl effect on cap5 genes but not vice versa, suggesting that arl functions upstream of $m g r A$ in activating capsule. These results therefore strongly indicate that $\operatorname{arl} R$ activates cap5 genes through $m g r A$. Interestingly, further experiments using gel-shift assays did not find evidence of ArlR binding to the $m g r A$ promoter or MgrA binding to the cap5 promoter (results not shown), suggesting that intermediary regulators exist upstream and downstream of $m g r A$ in the arlRS-mgrA-cap5 regulatory pathway.

It should be noted here that our quantitative RT-PCR results showing that mgrA did not affect arl expression are inconsistent with those reported from Ingavale et al. (2003) and Manna et al. (2004). In these two previous reports, MgrA was first shown to activate arl expression by direct binding to the arl promoter region (Ingavale et al., 2003) but was later reported to indirectly repress arl through $\operatorname{sarV}$ (Manna et al., 2004). Because these two studies used the same strain (the sigB-deficient RN6390) and the results are contradictory, it is difficult to draw a firm conclusion as to how mgrA regulates arl based on the studies. On the other hand, since the sigB-positive strain Newman was used in our studies, it is conceivable that the discrepancy between our results and those from the two reports could be explained by strain difference.

In this study, besides arl, we also identified five additional potential regulatory loci affecting capsule gene expression. 
Our preliminary data showed that two of these newly identified regulators, $s b c D C$ and $c l p C$, were also involved in capsule gene regulation. In addition to $m g r A$ and $a g r$, recent reports showed that sae and sigB were also involved in capsule regulation (Steinhuber et al., 2003; Bischoff et al., 2004). Taken together, these results indicate that capsule is under the regulation of a surprisingly large number of regulators. One interesting question to ask would be why capsule regulation is regulated by so many regulators. Capsular polysaccharide is a large molecule that resides on the surface of the bacteria. Because of its size, capsule anchored on the cell surface is an important defence shield for the bacteria to avoid host phagocytosis by masking the C3b deposited on the bacterial cell wall from the phagocytes (Cunnion et al., 2003; Karakawa et al., 1988; Thakker et al., 1998). However, for the same reason, it can also mask other surface components required for staphylococcal pathogenesis. Indeed, it has been shown that capsule impedes the initial attachment of $S$. aureus to endothelial cells by masking the adhesins (Pohlmann-Dietze, 2000). Thus, the organism needs to regulate capsule production according to requirements at various stages of the infection process. $S$. aureus can cause various types of infection at various sites of the host; it is therefore not unreasonable to postulate that capsule is regulated differently in different infections. The need for the organism to fine-tune capsule production under different conditions may explain why capsule is regulated by several regulators. The impressive number of regulatory genes involved in capsule regulation further supports the notion that virulence genes in S. aureus are regulated by a complex regulatory network.

\section{ACKNOWLEDGEMENTS}

The authors thank Connie Chen for excellent technical support. This work was supported by grants AI37027 and AI54607 from the National Institute of Allergy and Infectious Diseases.

\section{REFERENCES}

Bae, T., Banger, A. K., Wallace, A., Glass, E. M., Aslund, F., Schneewind, O. \& Missiakas, D. M. (2004). Staphylococcus aureus virulence genes identified by bursa aurealis mutagenesis and nematode killing. Proc Natl Acad Sci U S A 101, 12312-12317.

Bischoff, M., Dunman, P., Kormanec, J., Macapagal, D., Murphy, E., Mounts, W., Berger-Bächi, B. \& Projan, S. (2004). Microarray-based analysis of the Staphylococcus aureus $\sigma^{\mathrm{B}}$ regulons. J Bacteriol 186, 4085-4099.

Bronner, S., Monteil, H. \& Prevost, G. (2004). Regulation of virulence determinants in Staphylococcus aureus: complexity and applications. FEMS Microbiol Rev 28, 183-200.

Cheung, A. L., Bayer, A. S., Zhang, G., Gresham, H. \&, Xiong. Y. Q. (2004). Regulation of virulence determinants in vitro and in vivo in Staphylococcus aureus. FEMS Immunol Med Microbiol 40, 1-9.

Cocchiaro, J. L., Gomez, M. I., Risley, A., Solinga, R., Sordelli, D. O. \& Lee, J. C. (2006). Molecular characterization of the capsule locus from non-typeable Staphylococcus aureus. Mol Microbiol 59, 948-960.
Cunnion, K. M., Zhang, H. M. \& Frank, M. M. (2003). Availability of complement bound to Staphylococcus aureus to interact with membrane complement receptors influences efficiency of phagocytosis. Infect Immun 71, 656-662.

Fournier, B. \& Hooper, D. C. (2000). A new two-component regulatory system involved in adhesion, autolysis, and extracellular proteolytic activity of Staphylococcus aureus. J Bacteriol 182, 3955-3964.

Fournier, B., Klier, A. \& Rapoport, G. (2001). The two-component system ArlS-ArlR is a regulator of virulence gene expression in Staphylococcus aureus. Mol Microbiol 41, 247-261.

Herbert, S., Newell, S. W., Lee, C. Y., Wieland, K. P., Dassy, B., Fournier, J. M., Wolz, C. \& Döring, G. (2001). Regulation of Staphylococcus aureus type 5 and type 8 capsular polysaccharides by $\mathrm{CO}_{2}$. J Bacteriol 183, 4609-4613.

Ingavale, S. S., Van Wamel, W. \& Cheung, A. L. (2003). Characterization of RAT, an autolysis regulator in Staphylococcus aureus. Mol Microbiol 48, 1451-1466.

Karakawa, W. W., Sutton, A., Schneerson, R., Karpas, A. \& Vann, W. F. (1988). Capsular antibodies induce type-specific phagocytosis of capsulated Staphylococcus aureus by human polymorphonuclear leukocytes. Infect Immun 56, 1090-1095.

Klumpp, S. \& Krieglstein, J. (2002). Phosphorylation and dephosphorylation of histidine residues in proteins. Eur J Biochem 269, 1067-1071.

Kraemer, G. R. \& landolo, J. J. (1990). High-frequency transformation of Staphylococcus aureus by electroporation. Curr Microbiol 21, 373-376.

Kreiswirth, B. N., Lofdahl, S., Betley, M. J., O'Reilly, M., Shlievert, P. M., Bergdoll, M. S. \& Novick, R. P. (1983). The toxic shock syndrome exotoxin structural gene is not detectably transmitted by a prophage. Nature 305, 709-712.

Lee, C. Y. \& Lee, J. C. (2006). Staphylococcal capsules. In GramPositive Pathogens, 2nd edn, pp. 456-463. Edited by V. Fischetti, R. P. Novick, J. Ferretti, D. Portnoy \& J. Rood. Washington, DC: American Society for Microbiology.

Liang, X., Zheng, L., Landwehr, C., Lunsford, D., Holmes, D. \& Ji, Y. (2005). Global regulation of gene expression by ArlRS, a twocomponent signal transduction regulatory system of Staphylococcus aureus. J Bacteriol 187, 5486-5492.

Lin, W. S., Cunneen, T. \& Lee, C. Y. (1994). Sequence analysis and molecular characterization of genes required for the biosynthesis of type 1 capsular polysaccharide in Staphylococcus aureus. J Bacteriol 176, 7005-7016.

Luong, T. T. \& Lee, C. Y. (2002). Overproduction of type 8 capsular polysaccharide augments Staphylococcus aureus virulence. Infect Immun 70, 3389-3395.

Luong, T. T., Sau, S., Gomez, M., Lee, J. C. \& Lee, C. Y. (2002). Regulation of Staphylococcus aureus capsular polysaccharide expression by agr and sarA. Infect Immun 70, 444-450.

Luong, T. T., Newell, S. W. \& Lee, C. Y. (2003). $m g r$, a novel global regulator in Staphylococcus aurues. J Bacteriol 185, 3703-3710.

Luong, T. Y., Dunman, P. M., Murphy, E., Projan, S. J. \& Lee, C. Y. (2006). Transcriptional profiling of the mgrA regulon in Staphylococcus aureus. J Bacteriol 188, 1899-1910.

Manna, A. C., Ingavale, S. S., Maloney, M., van Wamel, W. \& Cheung, A. L. (2004). Identification of sarV (SA2062), a new transcriptional regulator, is repressed by SarA and MgrA (SA0641) and involved in the regulation of autolysis in Staphylococcus aureus. J Bacteriol 186, 5267-5280.

Novick, R. P. (2003). Autoinduction and signal transduction in the regulation of staphylococcal virulence. Mol Microbiol 48, 1429-1449. 
Novick, R. P., Edelman, I., Schwesinger, M. D., Gruss, A. D., Swanson, E. C. \& Pattee, P. A. (1979). Genetic translocation in Staphylococcus aureus. Proc Natl Acad Sci U S A 76, 400-404.

Novick, R. P., lordanescu, S., Projan, S. J., Kornblum, J. \& Edelman, I. (1989). pT181 plasmid replication is regulated by a countertranscript-driven transcriptional attenuator. Cell 59, 395-404.

O'Riordan, K. \& Lee, J. C. (2004). Staphylococcus aureus capsular polysaccharides. Clin Microbiol Rev 17, 218-234.

Ouyang, S., Sau, S. \& Lee, C. Y. (1999). Promoter analysis of the cap8 operon involved in type 8 capsular polysaccharide production in Staphylococcus aureus. J Bacteriol 181, 2492-2500.

Pohlmann-Dietze, P., Ulrich, M., Kiser, K. B., Doring, G., Lee, J. C., Fournier, J. M., Botzenhart, K. \& Wolz, C. (2000). Adherence of Staphylococcus aureus to endothelial cells: influence of the capsular polysaccharide, the global regulator $a g r$, and the bacterial growth phase. Infect Immun 68, 4865-4871.

Sau, S., Bhasin, N., Wann, E. R., Lee, J. C., Foster, T. J. \& Lee, C. Y. (1997a). The Staphylococcus aureus allelic genetic loci for serotype 5 and 8 capsule expression contain the type-specific genes flanked by common genes. Microbiology 143, 2395-2405.

Sau, S., Sun, J. \& Lee, C. Y. (1997b). Molecular characterization and transcriptional analysis of type 8 genes in Staphylococcus aureus. J Bacteriol 179, 1614-1621.
Steinhuber, A., Goerke, C., Bayer, M. G., Doring, G. \& Wolz, C. (2003). Molecular architecture of the regulatory locus sae of Staphylococcus aureus and its impact on expression of virulence factors. J Bacteriol 185, 6278-6286.

Thakker, M., Park, J.-S., Carey, V. \& Lee, J. C. (1998). Staphylococcus aureus serotype 5 capsular polysaccharide is antiphagocytic and enhances bacterial virulence in a murine bacteremia model. Infect Immun 66, 5183-5189.

Truong-Bolduc, Q. C., Zhang, X. \& Hooper, D. C. (2003). Characterization of NorR protein, a multifunctional regulator of norA expression in Staphylococcus aureus. J Bacteriol 185, 31273138.

Wang, P. Z., Projan, S. J., Leason, K. R. \& Novick, R. P. (1987). Translational fusion with a secretory enzyme as an indicator. J Bacteriol 169, 3082-3087.

Yansura, D. G. \& Henner, D. J. (1984). Use of Escherichia coli lac repressor and operator to control gene expression in Bacillus subtilis. Proc Natl Acad Sci U S A 81, 439-443.

Zukowski, M. M., Gaffiney, D. G., Speck, D., Kauffmann, M., Findeli, A., Wisecup, A. \& Lecocq, J. P. (1983). Chromogenic identification of genetic regulatory signals in Bacillus subtilis based on expression of a cloned Pseudomonas gene. Proc Natl Acad Sci U S A 80, 1101-1105. 